\title{
ON THE RADIUS OF CONVERGENCE OF SOME NEWTON-TYPE METHODS IN BANACH SPACES
}

\author{
IOANNIS K. ARGYROS ${ }^{a}$ AND SAÏD HILOUT ${ }^{b}$
}

\begin{abstract}
We determine the radius of convergence for some Newton-type methods (NTM) for approximating a locally unique solution of an equation in a Banach space setting. A comparison is given between the radii of (NTM) and Newton's method (NM). Numerical examples further validating the theoretical results are also provided in this study.
\end{abstract}

\section{INTRODUCTION}

In this study we are concerned with the problem of approximating a locally unique solution $x^{\star}$ of equation

$$
F(x)=0,
$$

where $F$ is a Fréchet-differentiable operator defined on a open convex subset $\mathcal{D}$ of a Banach space $\mathcal{X}$ with values in a Banach space $\mathcal{Y}$.

The field of computational sciences has seen a considerable development in mathematics, engineering sciences and economic equilibrium theory. For example, dynamic systems are mathematically modeled by difference or differential equations and their solutions usually represent the states of the systems. For the sake of simplicity, assume that a time-invariant system is driven by the equation $\dot{x}=T(x)$, for some suitable operator $T$, where $x$ is the state. Then the equilibrium states are determined by solving equation (1.1). Similar equations are used in the case of discrete systems. The unknowns of engineering equations can be functions (difference, differential and integral equations), vectors (systems of linear or nonlinear algebraic equations), or real or complex numbers (single algebraic equations with single unknowns). Except in special cases, the most commonly used solution methods are

Received by the editors September 22, 2010. Revised August 9, 2011. Accepted Aug. 12, 2011. 2000 Mathematics Subject Classification. 65G99, 65K10, 65B05, 47H17, 49M15.

Key words and phrases. Newton's method, Newton-type method, Banach space, local convergence, radius of convergence, Lipschitz-Hölder continuity. 
iterative-when starting from one or several initial approximations a sequence is constructed that converges to a solution of the equation. Iteration methods are also applied for solving optimization problems. In such cases, the iteration sequences converge to an optimal solution of the problem at hand. Since all of these methods have the same recursive structure, they can be introduced and discussed in a general framework. We note that in computational sciences, the practice of numerical analysis for finding such solutions is essentially connected to variants of Newton's method.

The most popular method for generating a sequence approximating $x^{\star}$ is undoubtedly the quadratically convergent Newton's method (NM)

$$
x_{n+1}=x_{n}-F^{\prime}\left(x_{n}\right)^{-1} F\left(x_{n}\right), \quad(n \geq 0), \quad\left(x_{0} \in \mathcal{D}\right) .
$$

Here, $F^{\prime}(x) \in \mathcal{L}(\mathcal{X}, \mathcal{Y}),(x \in \mathcal{D})$, the space of bounded linear operators from $\mathcal{X}$ into $\mathcal{Y}$ denotes the Fréchet-derivative of operator $F$ [3].

An other method for approximating $x^{\star}$ is the cubically convergent Newton-type method (NTM) given by

$$
\begin{aligned}
& y_{n}=x_{n}-F^{\prime}\left(x_{n}\right)^{-1} F\left(x_{n}\right), \quad(n \geq 0), \quad\left(x_{0} \in \mathcal{D}\right) \\
& x_{n+1}=y_{n}-F^{\prime}\left(x_{n}\right)^{-1} F\left(y_{n}\right) .
\end{aligned}
$$

(NM) requires the computation of inverse and one function evaluation at each step. There are few situations when one computes the inverse of a matrix. However, (NTM) requires the computation of one inverse and two functions evaluation. Note also that in the case of (NTM), one always computes the solution of a linear system (which is by far more easy to compute than the inverse). The local/semilocal convergence results for (NM) has been examined by many authors [1]-[4], [9]-[12]. The semilocal convergence of (NTM) has been studied in [2]-[7]. A survey of results on (NTM) is given in [3], [4] and the references there.

Here, we are interested in the local convergence of (NTM). Rheinboldt [11], Traub and Woźniakowsi [12] used the $L$-Lipschitz condition $(L>0)$ :

$$
\left\|F^{\prime}\left(x^{\star}\right)^{-1}\left(F^{\prime}(x)-F^{\prime}(y)\right)\right\| \leq L\|x-y\| \quad \text { for all } \quad x, y \in \mathcal{D}
$$

to provide the radius of convergence for (NM) given by

$$
r=\frac{2}{3 L} \text {. }
$$

In view of (1.4), there always exists $L_{0}>0$ such that

$$
\left\|F^{\prime}\left(x^{\star}\right)^{-1}\left(F^{\prime}(x)-F^{\prime}\left(x^{\star}\right)\right)\right\| \leq L_{0}\left\|x-x^{\star}\right\| \quad \text { for all } \quad x \in \mathcal{D} .
$$


Note that

$$
L_{0} \leq L
$$

holds in general and $\frac{L}{L_{0}}$ can be arbitrarily large [2]-[4]. Argyros [2], [3] used a combination of (1.4) and (1.5) to provide the radius of convergence of (NM) by the following

$$
r_{A}=\frac{2}{2 L_{0}+L}
$$

Note that

$$
r \leq r_{A} .
$$

Moreover, inequality (1.7) is strict if $L_{0}<L$. In [2], [3], Argyros provided a larger ball using the more precise (1.5) instead of (1.4) to provide a tighter upper bound on the norm $\left\|F^{\prime}(x)^{-1} F^{\prime}\left(x^{\star}\right)\right\|(x \in \mathcal{D})$. The same approach leads to tighter error bounds on the distances $\left\|x_{n+1}-x_{n}\right\|,\left\|x_{n}-x^{\star}\right\|(n \geq 0)$ and at least as precise information on the uniqueness of the solution $x^{\star}$.

The results under Lipschitz-type conditions are extended in the $p$-Hölder case $(p \in(0,1])$ by assuming

$$
\left\|F^{\prime}\left(x^{\star}\right)^{-1}\left(F^{\prime}(x)-F^{\prime}(y)\right)\right\| \leq L\|x-y\|^{p} \quad \text { for all } \quad x, y \in \mathcal{D}
$$

and

$$
\left\|F^{\prime}\left(x^{\star}\right)^{-1}\left(F^{\prime}(x)-F^{\prime}\left(x^{\star}\right)\right)\right\| \leq L_{0}\left\|x-x^{\star}\right\|^{p} \quad \text { for all } \quad x \in \mathcal{D} .
$$

If $p=1$, conditions (1.8) and (1.9) are reduced to (1.4) and (1.5), respectively. In the $p$-Hölder case, the radius of convergence ([2]-[4], [10]) is

$$
r_{p}=\left(\frac{1+p}{(1+p) L_{0}+L}\right)^{1 / p} .
$$

From now, we denote by

$$
U\left(x^{\star}, R\right)=\left\{x \in \mathcal{X}:\left\|x-x^{\star}\right\|<R\right\}
$$

the open ball centered at $x^{\star}$ and of radius $R>0$, whereas $\bar{U}\left(x^{\star}, R\right)$ denotes its closure. The paper is organized as follows: Section 2 contains the local convergence analysis of (NTM), whereas the applications are given in Section 3. 


\section{Local Convergence Analysis of (NTM)}

It is convenient for us to introduce some constants, functions and sequences.

In view of (1.6), there exists $c \geq 1$, such that

$$
L=c L_{0} .
$$

Define function $f_{c}$ on $[0, c]$ by

$$
f_{c}(t)=t\left(\left((1+p)\left(1-\frac{t}{c}\right)+t\right)^{1+p}-\left((1+p)\left(1-\frac{t}{c}\right)\right)^{1+p}\right)-(1+p)^{2+p}\left(1-\frac{t}{c}\right)^{3+p}
$$

for each fixed $c \geq 1$ and $p \in(0,1]$.

We have $f_{c}(0)=-(1+p)^{2+p}<0, f_{c}(c)=c^{2+p} \geq 1>0$ and $f_{c}$ is continuous on $[0, c]$. It follows from the intermediate value theorem that there exists a zero of function $f_{c}$ in $(0, c)$. Denote by $t^{c}$ the minimal zero of $f_{c}$ in $(0, c)$.

Clearly, we have

$$
t^{1} \leq t^{c} \quad \text { for all } \quad c \geq 1
$$

We need an Ostrowski-type representation for (NTM) [2]-[4].

Lemma 2.1. If (NTM) is well defined for all $n \geq 0, F\left(x^{\star}\right)=0$ and $F^{\prime}\left(x^{\star}\right)^{-1} \in$ $\mathcal{L}(\mathcal{Y}, \mathcal{X})$, then

$$
\begin{aligned}
& x_{n+1}-x^{\star} \\
& =\left(F^{\prime}\left(x^{\star}\right)^{-1} F^{\prime}\left(x_{n}\right)\right)^{-1} F^{\prime}\left(x^{\star}\right)^{-1} \int_{0}^{1}\left(F^{\prime}\left(x_{n}\right)-F^{\prime}\left(\theta y_{n}+(1-\theta) x^{\star}\right)\right) d \theta \times \\
& \left(F^{\prime}\left(x^{\star}\right)^{-1} F^{\prime}\left(x_{n}\right)\right)^{-1} F^{\prime}\left(x^{\star}\right)^{-1} \int_{0}^{1}\left(F^{\prime}\left(x_{n}\right)-F^{\prime}\left(\theta x_{n}+(1-\theta) x^{\star}\right)\right) d \theta\left(x_{n}-x^{\star}\right)
\end{aligned}
$$

holds for all $n \geq 0$.

Proof. Using (1.3) and Taylor's formula, we get in turn

$$
\begin{aligned}
& x_{n+1}-x^{\star} \\
& =y_{n}-x^{\star}-F^{\prime}\left(x_{n}\right)^{-1}\left(F\left(y_{n}\right)-F\left(x^{\star}\right)\right) \\
& =F^{\prime}\left(x_{n}\right)^{-1} \int_{0}^{1}\left(F^{\prime}\left(x_{n}\right)-F^{\prime}\left(\theta y_{n}+(1-\theta) x^{\star}\right)\right) d \theta\left(y_{n}-x^{\star}\right) \\
& =F^{\prime}\left(x_{n}\right)^{-1} \int_{0}^{1}\left(F^{\prime}\left(x_{n}\right)-F^{\prime}\left(\theta y_{n}+(1-\theta) x^{\star}\right)\right) d \theta\left(x_{n}-x^{\star}-F^{\prime}\left(x_{n}\right)^{-1} F\left(x_{n}\right)\right)
\end{aligned}
$$

and since

$$
\begin{aligned}
& x_{n}-x^{\star}-F^{\prime}\left(x_{n}\right)^{-1} F\left(x_{n}\right) \\
& =F^{\prime}\left(x_{n}\right)^{-1} \int_{0}^{1}\left(F^{\prime}\left(x_{n}\right)-F^{\prime}\left(\theta x_{n}+(1-\theta) x^{\star}\right)\right) d \theta\left(x_{n}-x^{\star}\right),
\end{aligned}
$$


we obtain (2.4). That completes the proof of Lemma 2.1.

We can show the following local convergence result for (NTM).

Theorem 2.2. Let $F: \mathcal{D} \subseteq \mathcal{X} \longrightarrow \mathcal{Y}$ be a Fréchet-differentiable operator. Let $x^{\star} \in \mathcal{D}$, such that $F\left(x^{\star}\right)=0$ and $F^{\prime}\left(x^{\star}\right)^{-1} \in \mathcal{L}(\mathcal{Y}, \mathcal{X})$. Let $p \in(0,1]$, such that $p$-Hölder conditions (1.8) and (1.9) hold. Denotes by $t^{c}$ the minimal zero of $f_{c}$ in $(0, c)$ and suppose $U\left(x^{\star}, r^{\star}\right) \subseteq \mathcal{D}$, where

$$
r^{\star}=\left(\frac{(1+p) c}{L}\right)^{1 / p}
$$

Then, sequence $\left\{x_{n}\right\}$ generated by (NTM) is well defined, remains in $\bar{U}\left(x^{\star}, r^{c}\right)$ for all $n \geq 0$, where

$$
r^{c}=\left(\frac{t^{c}}{L}\right)^{1 / p}
$$

and $\left\{x_{n}\right\}$ converges to $x^{\star} \in U\left(x^{\star}, r^{c}\right)$ solution of (1.1), provided that $x_{0} \in U\left(x^{\star}, r^{c}\right)$.

Moreover, for $e_{n}=\left\|x_{n}-x^{\star}\right\|(n \geq 0)$, the following estimate holds:

$$
e_{n+1} \leq a_{n+1},
$$

where, scalar iteration $\left\{a_{n}\right\}(n \geq 0)$ is given by

$$
a_{n+1}=\frac{L}{\left(1-\frac{L}{c} e_{n}^{p}\right)^{2}}\left(\left(1+\frac{L e_{n}^{p}}{(1+p)\left(1-\frac{L}{c} e_{n}^{p}\right)}\right)^{1+p}-1\right) \frac{e_{n}^{1+p}}{1+p} .
$$

Furthemore, the solution $x^{\star}$ is unique in $U\left(x^{\star}, r^{\star}\right)$.

Proof. Let $x \in U\left(x^{\star}, r_{0}=\left(\frac{c}{L}\right)^{1 / p}\right)$. Then, using (1.9), we obtain

$$
\left\|F^{\prime}\left(x^{\star}\right)^{-1}\left(F^{\prime}(x)-F^{\prime}\left(x^{\star}\right)\right)\right\| \leq L_{0}\left\|x-x^{\star}\right\|^{p}<\frac{L}{c} \frac{c}{L}=1 .
$$

It follows from (2.7) and the Banach Lemma of invertible operators [3], [4], [9], $F^{\prime}(x)^{-1} \in \mathcal{L}(\mathcal{Y}, \mathcal{X})$ and

$$
\left\|F^{\prime}(x)^{-1} F^{\prime}\left(x^{\star}\right)\right\| \leq \frac{1}{1-\frac{L}{c}\left\|x-x^{\star}\right\|^{p}} .
$$

By hypothesis $x_{0} \in U\left(x^{\star}, r^{c}\right) \subseteq U\left(x^{\star}, r_{0}\right)$. Hence, (2.8) for $x=x_{0}$ gives

$$
\left\|F^{\prime}\left(x_{0}\right)^{-1} F^{\prime}\left(x^{\star}\right)\right\| \leq \frac{1}{1-\frac{L}{c}\left\|x_{0}-x^{\star}\right\|^{p}} .
$$


Using (2.4) for $n=0,(1.8),(1.9)$ and (2.9), we get

$$
\begin{aligned}
\left\|x_{1}-x^{\star}\right\| & \leq \frac{L}{\left(1-\frac{L}{c} e_{0}^{p}\right)^{2}}\left(\left(1+\frac{L e_{0}^{p}}{(1+p)\left(1-\frac{L}{c} e_{0}^{p}\right)}\right)^{1+p}-1\right) \frac{e_{0}^{1+p}}{1+p} \\
& <\left\|x_{0}-x^{\star}\right\|,
\end{aligned}
$$

by the definition of $t^{c},(2.5)$ and the following estimates

$$
\begin{gathered}
\left\|F^{\prime}\left(x^{\star}\right)^{-1} \int_{0}^{1}\left(F^{\prime}\left(x_{0}\right)-F^{\prime}\left(\theta x_{0}+(1-\theta) x^{\star}\right)\right) d \theta\right\| \\
\leq L \int_{0}^{1}\left\|x_{0}-(1-\theta) x^{\star}-\theta x_{0}\right\| d \theta \\
=L\left\|x_{0}-x^{\star}\right\|^{p} \int_{0}^{1}(1-\theta)^{p} d \theta=\frac{L\left\|x_{0}-x^{\star}\right\|^{p}}{1+p}, \\
\left\|F^{\prime}\left(x^{\star}\right)^{-1} \int_{0}^{1}\left(F^{\prime}\left(x_{0}\right)-F^{\prime}\left(\theta y_{0}+(1-\theta) x^{\star}\right)\right) d \theta\right\| \\
\leq L \int_{0}^{1}\left\|x_{0}-(1-\theta) x^{\star}-\theta y_{0}\right\| d \theta, \\
x_{0}-(1-\theta) x^{\star}-\theta y_{0}=x_{0}-x^{\star}-\theta\left(y_{0}-x^{\star}\right) \\
=x_{0}-x^{\star}-\theta F^{\prime}\left(x_{0}\right)^{-1} \int_{0}^{1}\left(F^{\prime}\left(x_{0}\right)-F^{\prime}\left(\theta x_{0}+(1-\theta) x^{\star}\right) d \theta\right)\left(x_{0}-x^{\star}\right)
\end{gathered}
$$

and

$$
\begin{aligned}
& \left\|F^{\prime}\left(x^{\star}\right)^{-1} \int_{0}^{1}\left(F^{\prime}\left(x_{0}\right)-F^{\prime}\left(\theta y_{0}+(1-\theta) x^{\star}\right)\right) d \theta\right\| \\
& \leq L e_{0}^{p} \int_{0}^{1}\left(1+\frac{\theta L e_{0}^{p}}{(1+p)\left(1-\frac{L}{c} e_{0}^{p}\right)}\right)^{p} d \theta \\
& =\left(1-\frac{L}{c} e_{0}^{p}\right)\left(\left(1+\frac{L e_{0}^{p}}{(1+p)\left(1-\frac{L}{c} e_{0}^{p}\right)}\right)^{1+p}-1\right) .
\end{aligned}
$$

Estimate (2.10) implies that $x_{1} \in U\left(x^{\star}, r^{c}\right)$ and (2.6) holds for $n=0$. Let us assume (2.6) and $x_{k} \in U\left(x^{\star}, r^{c}\right)$ hold for all $k \leq n$. Then, as in (2.8)-(2.14), we have the estimates (with $x_{n}, y_{n}$ replacing $x_{0}$ and $y_{0}$, respectively):

$$
\begin{gathered}
\left\|F^{\prime}\left(x_{n}\right)^{-1} F^{\prime}\left(x^{\star}\right)\right\| \leq \frac{1}{1-\frac{L}{c} e_{n}^{p}}, \\
\left\|F^{\prime}\left(x^{\star}\right)^{-1} \int_{0}^{1}\left(F^{\prime}\left(x_{n}\right)-F^{\prime}\left(\theta x_{n}+(1-\theta) x^{\star}\right)\right) d \theta\right\| \leq \frac{L e_{n}^{p}}{1+p},
\end{gathered}
$$




$$
\begin{aligned}
& \left\|F^{\prime}\left(x^{\star}\right)^{-1} \int_{0}^{1}\left(F^{\prime}\left(x_{n}\right)-F^{\prime}\left(\theta y_{n}+(1-\theta) x^{\star}\right)\right) d \theta\right\| \\
& \leq\left(1-\frac{L}{c} e_{n}^{p}\right)\left(\left(1+\frac{L e_{n}^{p}}{(1+p)\left(1-\frac{L}{c} e_{n}^{p}\right)}\right)^{1+p}-1\right) .
\end{aligned}
$$

Using (2.4), we have

$$
\begin{aligned}
& \left\|x_{n+1}-x^{\star}\right\| \\
& \leq\left\|F^{\prime}\left(x_{n}\right)^{-1} F^{\prime}\left(x^{\star}\right)\right\|^{2} \int_{0}^{1}\left\|F^{\prime}\left(x^{\star}\right)^{-1}\left(F^{\prime}\left(x_{n}\right)-F^{\prime}\left(\theta y_{n}+(1-\theta) x^{\star}\right)\right)\right\| d \theta \\
& \quad \times \int_{0}^{1}\left\|F^{\prime}\left(x^{\star}\right)^{-1}\left(F^{\prime}\left(x_{n}\right)-F^{\prime}\left(\theta x_{n}+(1-\theta) x^{\star}\right)\right)\right\| d \theta\left\|x_{n}-x^{\star}\right\| .
\end{aligned}
$$

By (2.15)-(2.18), we obtain

$$
e_{n+1}=\left\|x_{n+1}-x^{\star}\right\| \leq a_{n+1}<e_{n},
$$

the induction is completed, $x_{n+1} \in U\left(x^{\star}, r^{c}\right)$ and $\left\{x_{n}\right\}$ converges to $x^{\star}$.

We shall finally show the uniqueness part. Let $y^{\star} \in U\left(x^{\star}, r^{\star}\right)$ be a solution of $F(x)=0$ and set

$$
\mathcal{Q}=\int_{0}^{1} F^{\prime}\left(y^{\star}+\theta\left(x^{\star}-y^{\star}\right)\right) d \theta .
$$

Using (2.4), we have in turn:

$$
\begin{aligned}
\left\|A\left(x^{\star}\right)^{-1}\left(\mathcal{Q}-F^{\prime}\left(x^{\star}\right)\right)\right\| & \leq\left\|F^{\prime}\left(x^{\star}\right)^{-1} \int_{0}^{1}\left(F^{\prime}\left(y^{\star}+\theta\left(x^{\star}-y^{\star}\right)\right)-F^{\prime}\left(x^{\star}\right)\right) d \theta\right\| \\
& \leq \frac{L}{c}\left\|x^{\star}-y^{\star}\right\|^{p} \int_{0}^{1} \theta^{p} d \theta \\
& =\frac{L}{c(1+p)}\left\|x^{\star}-y^{\star}\right\|^{p}<1 .
\end{aligned}
$$

It follows from (2.21) and the Banach Lemma on invertible operators that $\mathcal{Q}^{-1}$ exists. In view of the identity

$$
0=F\left(x^{\star}\right)-F\left(y^{\star}\right)=\mathcal{Q}\left(x^{\star}-y^{\star}\right)
$$

we conclude

$$
x^{\star}=y^{\star} .
$$

That completes the proof of Theorem 2.2. 
Remark 2.3. The condition $U\left(x^{\star}, r^{\star}\right) \subseteq \mathcal{D}$ is needed to show the uniqueness part. It can be replaced by

$$
U\left(x^{\star}, r_{0}\right) \subseteq \mathcal{D}
$$

However, we then have

$$
U\left(x^{\star}, r_{0}\right) \subseteq U\left(x^{\star}, r^{\star}\right) .
$$

Next, we shall show that (NTM) converges with order $1+2 p$.

Proposition 2.4. Under the hypotheses of Theorem 2.2, we have

$$
e_{n+1} \leq a_{n+1} \leq b_{n+1},
$$

where,

$$
b_{n+1}=\frac{L^{2}\left(1-\frac{L}{c} e_{n}^{p}\right)}{\left(1-\frac{L_{0}}{c} e_{n}^{p}\right)^{2}}\left(\frac{L}{1-\frac{L}{c} e_{n}^{p}}+\frac{p L e_{n}^{p}}{2(1+p)\left(1-\frac{L}{c} e_{n}^{p}\right)^{2}}\right) e_{n}^{1+2 p} .
$$

Proof. Define function $g_{n, c}$ on $[1, \beta]$, where,

$$
\beta=1+\frac{L e_{n}^{p}}{(1+p)\left(1-\frac{L}{c} e_{n}^{p}\right)}
$$

by

$$
g_{n, c}(t)=t^{1+p} .
$$

Then, we get $g_{n, c}^{\prime}(t)=(1+p) t^{p}$ and $g_{n, c}^{\prime \prime}(t)=(1+p) p t^{p-1}$.

Taylor's formula guarantees the existence of $\gamma \in[1, \beta]$, such that

$$
\begin{aligned}
g_{n, c}(\beta)-g_{n, c}(1) & =g_{n, c}^{\prime}(\beta-1)+\frac{g_{n, c}^{\prime \prime}(\gamma)}{2}(\beta-1)^{2} \\
& =\frac{L e_{n}^{p}}{1-\frac{L}{c} e_{n}^{p}}+\frac{p L^{2} e_{n}^{2 p}}{2 \gamma^{1-p}(1+p)\left(1-\frac{L}{c} e_{n}^{p}\right)^{2}} \\
& \leq L\left(\frac{L}{1-\frac{L}{c} e_{n}^{p}}+\frac{p L e_{n}^{p}}{2(1+p)\left(1-\frac{L}{c} e_{n}^{p}\right)^{2}}\right) e_{n}^{p},
\end{aligned}
$$

since,

$$
\frac{1}{\gamma^{1-p}} \leq 1 \quad \text { for all } \gamma \in[1, \beta] .
$$

By the defintion of sequences $\left\{a_{n}\right\},\left\{b_{n}\right\}$ and (2.24), we obtain

$$
a_{n+1} \leq b_{n+1} \text { for all } n \geq 0 .
$$

That completes the proof of Proposition 2.4. 
Remark 2.5. The results obtained in this study can be applied for operators $F$ satisfying autonomous differential equations of the form

$$
F^{\prime}(x)=\mathcal{P}(F(x)),
$$

where, $\mathcal{P}: \mathcal{Y} \longrightarrow \mathcal{X}$ is a given continuous operator. As $F^{\prime}\left(x^{\star}\right)=\mathcal{P}\left(F\left(x^{\star}\right)\right)=P(0)$, we do not need to known $x^{\star}$ to verify conditions (1.5), (1.6), (1.7) and (1.8).

Let $F(x)=e^{x}-1$. Define $\mathcal{P}$ by $\mathcal{P}(x)=x+1$, then, (2.25) is satisfied (see also Example 3.1). Other applications can be found in [1]-[11].

\section{Applications}

Example 3.1 ([2]-[4]). Let $\mathcal{X}=\mathcal{Y}=\mathbb{R}$. Define function $F$ on $\mathcal{D}=[-1,1]$, given by

$$
F(x)=e^{x}-1 .
$$

Then, for $x^{\star}=0$, using (3.1), we have $F\left(x^{\star}\right)=0$ and $F^{\prime}\left(x^{\star}\right)=e^{0}=1$. Moreover, (1.8) and (1.9) hold for $p=1, L=e>L_{0}=e-1$, whereas $c=\frac{e}{e-1}=1.581976707$. Moreover, we have

$$
\begin{gathered}
f_{c}(t)=t\left(\left(2\left(1-\frac{(e-1) t}{e}\right)+t\right)^{2}-4\left(1-\frac{(e-1) t}{e}\right)^{2}\right)-8\left(1-\frac{(e-1) t}{e}\right)^{4}, \\
t^{c}=.6098166918, \quad r_{0}=\left(\frac{c}{L}\right)^{1 / p}=.5819767070 \\
r^{c}=\left(\frac{t^{c}}{L}\right)^{1 / p}=.2571658439, \quad r^{\star}=1.163953414 .
\end{gathered}
$$

Note that

$$
r^{c}=.2571658439<r_{A}=\frac{2}{2 L_{0}+L}=.3249472314 .
$$

Example 3.2 ([2]-[4]). Let $\mathcal{X}=\mathcal{Y}=\mathbb{R}$. Define function $F$ on $\mathcal{D}=[1,3]$, given by

$$
F(x)=\frac{2}{3} x^{3 / 2}-x
$$

Then, the zero of $F$ is $x^{\star}=\frac{9}{4}=2.25$. Using $(3.2), F^{\prime}\left(x^{\star}\right)=.5, L=2>L_{0}=1$, $c=2$ and $p=.5$. Moreover, we have

$$
\begin{gathered}
f_{c}(t)=t\left(\left(\frac{3}{2}\left(1-\frac{t}{2}\right)+t\right)^{\frac{3}{2}}-\left(\frac{3}{2}\left(1-\frac{t}{2}\right)\right)^{\frac{3}{2}}\right)-\left(\frac{3}{2}\right)^{\frac{5}{2}}\left(1-\frac{t}{2}\right)^{\frac{7}{2}}, \\
t^{c}=.6412553390, \quad r_{0}=1, \\
r^{c}=.5662399399, \quad r^{\star}=1.224744871 .
\end{gathered}
$$

Note that 


$$
r^{c}=.5662399399<r_{A}=\left(\frac{1+p}{(1+p) L_{0}+L}\right)^{1 / p}=.6546536707 .
$$

Example 3.3. Let $\mathcal{X}=\mathcal{Y}=\mathcal{C}[0,1]$, the space of continuous functions defined on $[0,1]$, equipped with the max norm and $\mathcal{D}=\bar{U}(0,1)$. Define function $F$ on $\mathcal{D}$, given by

$$
F(h)(x)=h(x)-5 \int_{0}^{1} x \theta h(\theta)^{3} d \theta .
$$

Then, we have:

$$
\begin{aligned}
& f_{c}(t)=t\left(\left(2\left(1-\frac{t}{2}\right)+t\right)^{2}-4\left(1-\frac{t}{2}\right)^{2}\right)-8\left(1-\frac{t}{2}\right)^{4}, \\
& F^{\prime}(h[u])(x)=u(x)-15 \int_{0}^{1} x \theta h(\theta)^{2} u(\theta) d \theta \quad \text { for all } u \in \mathcal{D} .
\end{aligned}
$$

Using (3.3), (1.5), (1.6) for $x^{\star}(x)=0$ for all $x \in[0,1]$, we get

$$
\begin{array}{cc}
p=1, \quad L=15, \quad L_{0}=7.5, \quad c=2, \\
t^{c}=.6780149362, & r_{0}=.1333333333, \\
r^{c}=.04520099575, & r^{\star}=.2666666667 .
\end{array}
$$

Note that

$$
r^{c}=.04520099575<r_{A}=\frac{2}{2 L_{0}+L}=.06666666667 .
$$

Example 3.4. In the following Tables, we tabulate values of $t^{c}$ for $c=1, \frac{e}{e-1}=$ 1.581976707, 2 and $p=.1, .2, \cdots, 1$. We use Maple 13 for different calculations.

Comparison table (I)

\begin{tabular}{|r|l|l|l|l|}
\hline $\mathrm{p}$ & .1 & .2 & .3 & .4 \\
\hline$t^{1}$ & .4368349792 & .4429562040 & .4486068930 & .4538529047 \\
$t^{1.581976707}$ & .5466960852 & .5556998369 & .5640448368 & .571820981 \\
$t^{2}$ & .6027543663 & .6134232216 & .6233312759 & .6325810531 \\
\hline
\end{tabular}

Comparison table (II)

\begin{tabular}{|r|l|l|l|l|}
\hline $\mathrm{p}$ & .5 & .6 & .7 & .8 \\
\hline$t^{1}$ & .4587471202 & .4633326601 & .4676451584 & .4717144081 \\
$t^{1.581976707}$ & .5791007272 & .5859433703 & .5923980761 & .5985060824 \\
$t^{2}$ & .6412553390 & .6494219962 & .6571373857 & .6644488527 \\
\hline
\end{tabular}

Comparison table (III)

\begin{tabular}{|r|l|l|}
\hline $\mathrm{p}$ & .9 & 1 \\
\hline$t^{1}$ & .4755655764 & .4792201186 \\
$t^{1.581976707}$ & .6043023300 & .6098166918 \\
$t^{2}$ & .6713965712 & .6780149362 \\
\hline
\end{tabular}



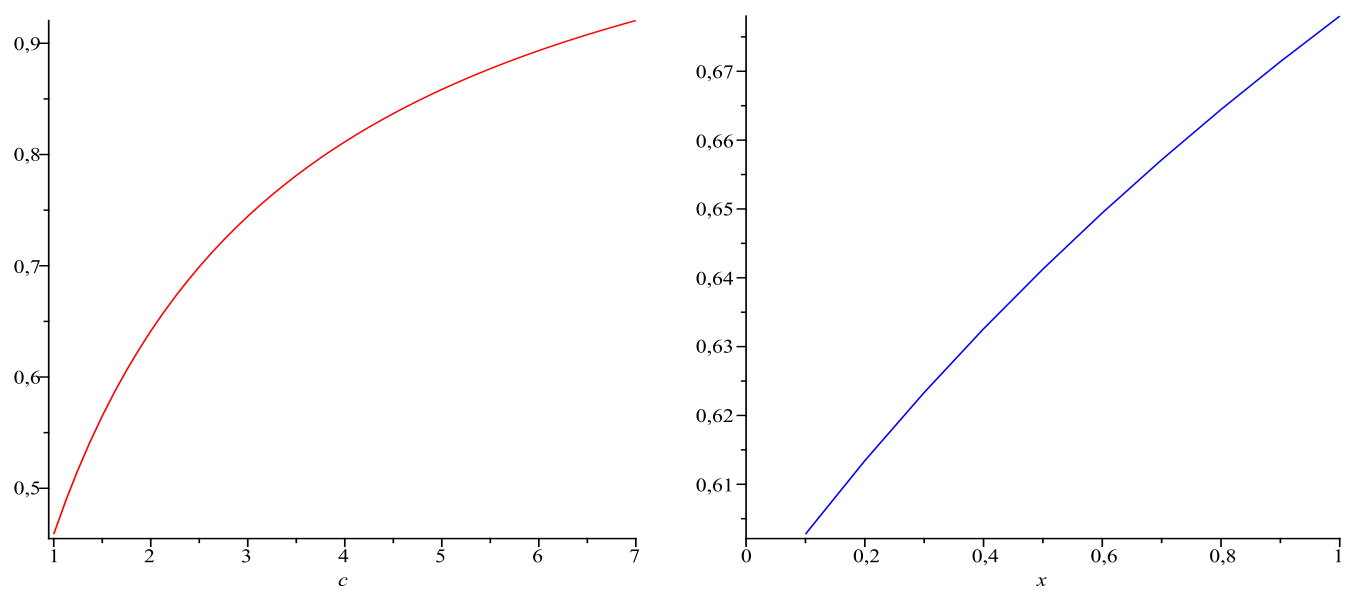

Figure 1. Left: Function $\varphi: c \longrightarrow t^{c}$ on [1;7]; Right: Function $\phi: p \longrightarrow$ $t^{c}$ on $[.1 ; 1]$.

It follows from Tables (I)-(III) that $t^{c}$ increses with $c$ and $p$. For also validating this result, see Fig. 1-left figure (for $p=.5$ and $c \in[1 ; 7]$ ) and fig. 1-right figure (for $c=2$ and $p \in[.1 ; 1])$. Since we can not have an explicit solution $t^{2}$ in terms of $p$ for function $\phi$ (Fig. 1-right figure), we simply plot the function $\phi$ from the list of points given by tables (I)-(III) for $c=2$.

\section{Conclusion}

We are interested in the radius of convergence for (NTM) in order to approximate a locally unique solution of an nonlinear equation in a Banach space setting. Using a combination of Hölder and center-Hölder conditions, we presented a comparison study between the radii of (NTM) and (NM). Applications and numerical examples are also provided in this study.

\section{REFERENCES}

1. Argyros, I.K.: On the radius of convergence of Newton's method. Intern. J. Comput. Math. 77 (2001), 389-400.

2. __ : A unifying local-semilocal convergence analysis and applications for two-point Newton-like methods in Banach space. J. Math. Anal. Appl. 298 (2004), 374-397.

3.___ Convergence and applications of Newton-type iterations. Springer-Verlag, 2008, New York.

4. Argyros, I.K. \& Hilout, S.: Efficient methods for solving equations and variational inequalities. Polimetrica Publisher, Milano, Italy, 2009. 
5. Ezquerro, J.A., Hernández, M.A. \& Salanova, M.A.: A discretization scheme for some conservative problems. Proceedings of the 8th Inter. Congress on Comput. and Appl. Math., ICCAM-98 (Leuven), J. Comput. Appl. Math. 115 (2000), 181-192.

6 . : A Newton-like method for solving some boundary value problems. Numer. Funct. Anal. Optimiz. 23 (2002), 791-805.

7. Gupta, D.K. \& Parhi, S.K.: A third order method for fixed points in Banach spaces. J. Math. Anal. Appl. 359 (2009), 642-652.

8. Hernández, M.A. \& Romero, N.: On a characterization of some Newton-like methods of $R$-order at least three. J. Comput. Appl. Math. 183 (2005), 53-66.

9. Kantorovich, L.V. \& Akilov, G.P.: Functional Analysis in normed spaces, Pergamon Press, Oxford, 1982.

10. Proinov, P.D.: General local convergence theory for a class of iterative processes and its applications to Newton's method. J. Complexity 25 (2009), 38-62.

11. Rheinboldt, W.C.: An adaptative continuation process for solving systems of nonlinear equations. Polish Acad. of Sciences, Banach Ctr. Publ. 3 (1977), 129-142.

12. Traub, J.F. \& Woźniakowsi, H.: Convergence and complexity of Newton iteration for operator equations. J. Assoc. Comput. Mach. 26 (1979), 250-258.

${ }^{a}$ Cameron university, Department of Mathematics Sciences, Lawton, OK 73505, USA

Email address: iargyros@cameron.edu

${ }^{\text {b} P o i t i e r s ~ u n i v e r s i t y, ~ L a b o r a t o i r e ~ d e ~ M a t h e ́ m a t i q u e s ~ e t ~ A p p l i c a t i o n s, ~ B d . ~ P i e r r e ~ e t ~}$ Marie Curie, Téléport 2, B.P. 30179, 86962 Futuroscope Chasseneuil Cedex, France

Email address: said.hilout@math.univ-poitiers.fr 